\title{
Clinical Data on Acute Kidney Injury Caused by Acute Organophosphorus Pesticide Poisoning
}

\author{
Hu Feng ${ }^{1}$, Wujian Peng ${ }^{1}$, Chen $\mathrm{Jie}^{2}$, Du Han ${ }^{2}$, Zhang He ${ }^{2}$, Jianrong Huang ${ }^{1}$, Xiaona Shi ${ }^{1}$, \\ Hanhan Kong ${ }^{1}$, Guixia Li $^{1}$ \\ ${ }^{1}$ Center for Kindey Disease, $2^{\text {nd }}$ Affiliated Hospital, Southern University of Sciences and Technology, Shenzhen, China \\ ${ }^{2}$ Division of Nephrology Center, The $989^{\text {th }}$ Hospital, Luoyang, China
}

Email address:

Jfjhf1504@126.com (Hu Feng)

\section{To cite this article:}

Hu Feng, Wujian Peng, Chen Jie, Du Han, Zhang He, Jianrong Huang, Xiaona Shi, Hanhan Kong, Guixia Li. Clinical Data on Acute Kidney Injury Caused by Acute Organophosphorus Pesticide Poisoning. American Journal of Clinical and Experimental Medicine.

Vol. 9, No. 5, 2021, pp. 135-141. doi: 10.11648/j.ajcem.20210905.11

Received: February 23, 2021; Accepted: March 12, 2021; Published: September 10, 2021

\begin{abstract}
Background: Organophosphorus pesticides (OPs) are widely used in China and some developed countries because they are powerful pesticides with minimal damage to crops. However, OPs are highly toxic, especially to the human lungs, heart, brain, kidneys. Research on the pathogenesis and risk factors of acute kidney injury (AKI) caused by OP poisoning is limited, Researches which tend to have small sample sizes and are commonly case reports. Objective: This study aims to investigate the pathogenesis and risk factors of AKI caused by acute organophosphorus poisoning syndrome (AOPP), in order to enable the adoption of early and reasonable treatment methods, to improve the prognosis of patients. Methods: Retrospective analysis was performed on the laboratory findings of 275 patients with acute OP poisoningl from January 2009 to June 2016. (1) The 275 patients with acute OP poisoning were grouped according to the presence or absence of AKI, and testing was performed for statistical differences in the relevant clinical indicators between the two groups. (2) Univariate analysis was performed to compare the differences in these indicators between the two groups, and indicators with statistically significant differences included in logistic regression and other methods to analyze the risk factors affecting AKI. Results: Among the 275 patients with acute OP poisoning, 92 (33.5\%) had AKI. The cure rate of the AKI group was $69.6 \%$ and case fatality rate was $1.1 \%$ (1/92); the cure rate of the non-AKI group was $73.8 \%(135 / 183)$ and the case fatality rate was $0.5 \%(1 / 135)$; the difference in mortality rate between the two groups was not statistically significant $(\mathrm{Z}=0.94, \mathrm{P}=0.34)$. Comparison of general information and clinical characteristics revealed that only the mean C-reactive protein (CRP) and SCr levels differed significantly between the two groups $\left(\mathrm{Z}=6.55, \mathrm{P}<0.001 ; \mathrm{t}^{\prime}=-3.14, \mathrm{P}=0.002\right)$. Multivariate logistic regression analysis showed that the time of poisoning, time to blood purification therapy and CRP level were factors influencing the onset of AKI. The relationship between CRP level and logitP(AKI=1) was non-linear, and so no interpretation was made on the OR size. Conclusion: 1. The rate of AKI about acute OP poisoning is $54.4 \%$, Both glomeruli and tubules were involved. 2. The prognosis of patients with AKI is relatively poor. 3. The risk factors influencing AKI caused acute OP poisoning may be related to prolonged time of poisoning, prolonged time to blood purification therapy and high CRP level.
\end{abstract}

Keywords: Acute Organophosphorus Poisoning, Acute Kidney Injury, C-reactive Protein

\section{Introduction}

Organophosphorus pesticides (OPs) are acid ester compounds that are typically brown oily liquids, but a few can be found as crystals. They are easily decomposed when exposed to alkaline substances, and emit a garlic odor. Common formulations include emulsions, oils and powders, and they can be used as insecticides, fungicides, and herbicides. OPs can be classified according to their toxicity into extremely toxic, highly toxic, moderately toxic, and mildly toxic. Commonly used varieties include phorate, dichlorvos, and omethoate. As a chemical substance, 
organophosphorus compounds are widely used in different countries and regions for its efficient insecticidal and herbicidal effects $[1,2]$. According to incomplete statistics, OPs now form the class of pesticides with the highest level of production and usage in the world. However, given the current widespread use of OPs, its toxicity to mammals has been garnering greater attention. In the process of production and daily living, people may come into contact with OPs for various reasons such as aging production equipment, poorly sealed containers, damaged protective equipment, etc., which can cause the poisons to be directly or indirectly absorbed into the bloodstream from the respiratory tract, digestive tract, skin or mucous membranes, resulting in the corresponding toxic side effects. Another cause of organophosphorus poisoning is suicide. According to the World Health Organization, about 3 million people suffer from organophosphorus poisoning each year, which in turn can lead to death from multiple organ failure, including neurological, respiratory, cardiovascular and renal failure [3]. Of the 300,000 deaths related to organophosphorus poisoning, more than 250,000 deaths are caused by suicide, accounting for $1 / 3$ of global suicides. Furthermore, in a survey on disease morbidity and mortality, it was found that poisoning ranked second among injuries in the United States [4].

As a major agricultural country, China accounts for about $33 \%-35 \%$ of the world's annual production of organophosphorus. As a class of compounds in the extremely toxic and highly toxic categories, the resulting social security issues have become a widespread concern [5]. However, cases of acute poisoning are fairly complex, with rapid changes in the patients' condition that often seriously endanger their lives $[4,6]$. Some studies have found that in a cholinergic crisis [7-9], the kidney is directly damaged by acute organophosphorus poisoning, and once the disease involves the renal system, the patient's prognosis is often poor. In this paper, we will focus on exploring the pathophysiological changes involved in acute kidney injury (AKI) caused by acute organophosphorus poisoning, in order to identify risk factors for the early detection of AKI, which will ensure the implementation of reasonable treatment plans and improve the prognosis of patients.

Previous reports suggest that OP-induced AKI presents with various clinical manifestations. For example, mild cases may simply have abnormal urinalysis with positive findings for protein or (and) occult blood, whereas severe cases may present with rapidly developing oliguria/anuria, and even require renal replacement therapy [10-12]. Therefore, in order to further analyze and understand the clinical characteristics of OP-induced AKI, and to identify the risk factors for AKI, we collected and collated the clinical data of 275 patients with acute OP poisoning, who were admitted and treated in our department between January 2009 and June 2016. Based on which, we identified the risk factors for the susceptibility to AKI, in order to enable early prevention and treatment, thereby improving the prognosis of patients.

\section{Data and Methods}

\subsection{General Information}

\subsubsection{Patients}

We collected the clinical data of patients with acute OP poisoning, who were admitted to the nephrology blood purification center between January 2009 and June 2016. The observation form included items such as the patient's detailed medical history and basic information, time and type of poisoning, admission treatment plan, post-discharge outcome, and summary of key clinical treatments. Each observation form received was checked, and items in doubt were promptly followed up by reviewing the medical records and verifying with relevant staff. All parts with errors and omissions were corrected or filled in.

\subsubsection{Inclusion Criteria}

1. Patients with a clear history of OP exposure and presented with clinical features for acute organophosphorus poisoning syndrome (AOPP).

2. Patients who received adequate gastric lavage within 30 min-24 h after poisoning, and were only administered gastric lavage and cathartics prior to consultation.

3. Patients with complete clinical information.

4. Upon admission, all patients were aggressively treated with routine emergency measures, such as gastric lavage and cathartics, while actively excluding the risk of gastrointestinal bleeding.

\subsubsection{Exclusion Criteria}

1. Patients with a history of severe cardiovascular, liver, and chronic kidney disease, or diseases of other vital organs.

2. Patients in whom concomitant poisoning by other substances cannot be excluded.

3. Patients who failed to cooperate with treatment and refused rescue procedures after admission.

4. Patients with incomplete data and lack of case information.

5. Patients who were lost to follow-up.

\subsubsection{Relevant Diagnostic Criteria for $A O P P$}

Graded diagnostic criteria: with reference to Critical Care Medicine [13].

(1) Mild: Whole blood cholinesterase activity decreased to $70 \%-50 \%$ of the normal range, which may manifest as dizziness, headache, nausea, vomiting, sweating, chest tightness, blurred vision, and pupil narrowing.

(2) Moderate: Whole blood cholinesterase activity decreased to $50 \%-30 \%$ of the normal range, and in addition to more prominent M-like symptoms described above, patients also present with fasciculation, staggering gait, and confusion.

(3) Severe: Whole blood cholinesterase activity decreased to less than $30 \%$ of the normal range, as well as coma, pulmonary edema and respiratory failure on top of moderate clinical symptoms. 


\subsubsection{Diagnostic Criteria for AKI}

Diagnosis was based on the Kidney Disease: Improving Global Outcomes (KDIGO) recommendations, and can be made if one of the following conditions was met [14]: (1) Rapid decline in renal function, accumulation of metabolites in the body, disturbances of water, electrolyte and acid-base balance, rise in blood creatinine $\geq 25.2 \mathrm{umol} / \mathrm{L}$ or $50 \%$ increase from baseline level within $48 \mathrm{~h}$, and/or urine output $<$ $0.5 \mathrm{ml} /(\mathrm{kg} . \mathrm{h}$ ) for $>6 \mathrm{~h}$ (excluding obstructive nephropathy or dehydration). Patients were then staged according to absolute serum creatinine (SCr): Stage 1: absolute increase of $\geq 26.4$ umol/L or increase to $150 \%-200 \%$ of baseline. Stage $2: \mathrm{SCr}$ increased to $200 \%-300 \%$ of baseline. Stage 3: SCr increased to more than $300 \%$ of baseline or $\mathrm{SCr} \geq 354 \mathrm{umol} / \mathrm{L}$ with an acute increase of $44 \mathrm{umol} / \mathrm{L}$. Reference was made to the Chronic Kidney Disease (CKD) EPI \& MDRD GFR Calculator-(With SI Units) method for the calculation of glomerular filtration rate.

\subsection{Method}

\subsubsection{Data Collection}

We enrolled 287 patients with acute OP poisoning who were admitted to our hospital. All clinical information and data were collected after poisoning and during their hospital stay. Unified testing index standards and measurement units were used, and two people were assigned to collate and record the clinical data of the observed patients. The patients were screened according to the inclusion and exclusion criteria to obtain 275 cases that met the requirements of the study. Their prognosis was recorded separately according to their general condition, including gender, age, organophosphorus type, amount of poison, time of gastric lavage, type of blood purification therapy and whether multiple purification modalities were needed, time of AKI onset, peripheral blood C-reactive protein (CRP), blood leukocytes, serum creatinine ( $\mathrm{SCr}$ ), number of cases with hematuria/proteinuria, time of hematuria onset, time of proteinuria onset, and time from poisoning to the start of blood purification. In particular, the interval between the patient's exposure to the poison and the first gastric lavage was considered the time to gastric lavage. The interval between the time of poisoning and the start of blood purification therapy was considered the time to blood purification therapy.

\subsubsection{Treatment Methods}

\section{(i). Routine Medical Treatment}

Primary treatment: Patients were rapidly removed from the dangerous environment, contaminated clothing was removed, and contaminated body parts were cleaned using soap and water. If the eyes were exposed to the poison, they were rinsed with normal saline or water to prevent secondary absorption from damaging local tissues. The specific procedures were as follows.

Gastric lavage: Gastric lavage was actively administered to all patients admitted with OP poisoning after acute gastrointestinal bleeding was excluded. Patients were first induced to expel as much poison as possible (e.g. by inducing emesis) before gastric lavage was performed, i.e., first out and then in. During the operation, speed was key, and the pace of poison expulsion was accelerated to reduce absorption. Patients were placed in the left lateral decubitus position, with the head turned to the side. The total amount of gastric lavage solution was no less than $2000-5000 \mathrm{ml}$, and the temperature was maintained at the body temperature. The lavage was repeated with saline until the returning gastric fluid was clear and odorless. The gastric tube was retained after the first lavage, and multiple lavages could be performed according to the severity and changes of the patient's condition. The interval was about once/(1-2h) or once/(3-4h). For patients in critical condition, continuous gastric tube drainage and gastrointestinal decompression were administered.

Induced emesis: The patient's state of consciousness was determined upon admission. Early induced emesis was encouraged for patients who had orally ingested the poison and were conscious. The patient's posture was adjusted to ensure the lateral position of the head. The vomiting reflex was induced by touching the patient's posterior linguopharyngeal wall and tongue root with blunt-headed objects such as fingers and tongue depressors to accelerate the expulsion of the poison. If this failed, the patient may be given several drinks of warm water, and stimulation was repeated to promote poison expulsion. For patients who were in a coma, this method was not adopted, and gastric lavage was actively performed instead, as they were susceptible to aspiration pneumonia.

Catharsis. Gastric lavage can help to eliminate residual poisons in the esophagus and stomach, but is ineffective for components that have already been absorbed into the body. Intestinal enema and catharsis can effectively inhibit the further absorption of poisons by intestinal mucosal cells, and have a good effect on poisons that have been absorbed into the body but not into the blood. Reducing the amount of organophosphorus in the body can further diminish the inhibitory effect on cholinesterase, and hence relieve the symptoms of poisoning.

Activated Carbon Adsorption: Activated carbon is a broad-spectrum adsorbent that is porous, has a high surface density of particulate matter, and has a low level of irritation to the human body. It can assist in the removal of many drugs and poisons, and can block absorption. However, activated carbon has its limitations. As it is time dependent [15-17], activated carbon was actively administered only to patients who had been poisoned within an hour.

\section{(ii). Application of Specific Antidotes}

The main mechanism of action in organophosphorus poisoning is the inhibition of cholinesterase activity, which prevents the further breakdown of choline-based esters into choline and acetate [18], thereby exerting its toxic effects by affecting the termination of signal transduction at cholinergic synapses. Patients admitted to our hospital were actively administered cholinergic receptor antagonists to maintain a certain state of atropinization; patients were also administered the cholinesterase reactivator, pralidoxime chloride. We administered the conventional doses formulated according to 
China's national requirements: Mild cases: $0.5-0.75 \mathrm{~g}$ via intramuscular injection or intravenous infusion, re-administration not needed after thorough gastric lavage. Moderate cases: $0.75-1.5 \mathrm{~g}$ via intramuscular injection or intravenous infusion, often repeated 1 to 2 times. Severe cases: 1.5-2.0g via intramuscular injection or intravenous infusion, can be re-administered multiple times if, after waiting for half an hour to an hour, the patient still showed no clinical signs of sufficient drug administration [19]. Discontinuation of the drug was usually considered when clinical symptoms of poisoning disappeared and blood cholinesterase activity had increased to more than $50 \%$ in blood samples.

\section{(iii). Blood Purification Therapy}

Despite the many treatment methods listed above for patients with OP poisoning, the prognosis for patients is mostly unsatisfactory. Blood purification involves drawing blood out of the body and removing toxins or harmful substances from the blood through a device, in order relieve symptoms. The common methods of renal replacement therapy include hemodialysis, ultrafiltration, hemoperfusion, etc. In China, hemoperfusion is the most commonly used method, and should be performed as early as possible when conditions and indications are permit [20]. In this study, 209 patients received blood purification therapy within 1-24 h after poisoning. Those who ingested a large amount of poison, were in serious condition, and had multiple organ failure were treated with multiple modalities of blood purification.

\section{(iv). Symptomatic and Supportive Care}

Patients with shortness of breath and respiratory failure were given continuous oxygen inhalation, and mechanical ventilation (endotracheal intubation, ventilator application) if necessary, to assist their breathing. Patients were also given fluids to improve microcirculation, correct acidosis, and actively fight infection, as well as nutritional support treatment.

\section{Statistical Methods}

Analysis was performed using the SPSS 22 statistical software. Among the quantitative variables, those that followed a normal distribution were expressed as $\mathrm{S}$; between-group comparison was performed using the $t$-test, or the $t^{\prime}$ test for unequal variance. Quantitative variables that were not normally distributed were expressed as median and interquartile range $(\mathrm{M}(\mathrm{Q}))$, and between-group comparison was performed using the Wilcoxon rank sum test. Qualitative variables were expressed as frequencies and their rates or composition ratios; between-group comparison for gender was performed using the test, and between-group comparison for prognosis was performed using the Wilcoxon rank sum test. Multivariate logistic regression was performed to analyze the factors influencing the occurrence of AKI, and variables considered to be significant in the field were included in the analysis. Hypothesis testing of the independent variables was performed using Wald $x^{2}$ and the goodness of fit of the model was evaluated using the size of the likelihood function. For non-linear relationships between the independent variables and $\operatorname{logit} P(\mathrm{AKI}=1)$, the independent variables and their squares were included simultaneously into the model. The significance level was 0.05 (two-sided) for all.

\section{Results}

\subsection{General Information and Clinical Features of Systemic Damage in Patients with Acute OP Poisoning}

As indicated by the clinical characteristics of the 275 patients with acute OP poisoning, 92 (33.5\%) had AKI and $183(66.5 \%)$ did not; the mean age was $36.8 \pm 17.5$ years, with 109 males and 106 females; the mean time of poisoning was $4.0(3.0) \mathrm{h}$, the mean amount of poison was $50.0(75.0) \mathrm{ml}$, the mean time to gastric lavage was $2.0(2.5) \mathrm{h}$, the mean time to AKI was $12.3(17.0) \mathrm{h}$, the mean CRP level was $4.6(8.0)$ $\mathrm{mg} / \mathrm{L}$, the mean peripheral blood leukocyte was $14.7 \pm 6.5$ $10^{9} / \mathrm{L}$, the mean $\mathrm{SCr}$ was $65.8 \pm 22.0 \mathrm{umol} / \mathrm{L}$, and the mean time from poisoning to blood purification was $6.0(3.5) \mathrm{h}$. In the AKI group, the mean age was $38.2 \pm 17.7$ years, with 42 males and 50 females; the mean time of poisoning was 4.0 (3.0) $\mathrm{h}$, the mean amount of poison was 60.0 (80.0) $\mathrm{ml}$, the mean time to gastric lavage was $2.0(2.5) \mathrm{h}$, the mean time to AKI was 12.3 (17.0) $\mathrm{h}$, the mean CRP level was 8.0 (9.0) $\mathrm{mg} / \mathrm{L}$, the mean peripheral blood leukocyte was $15.2 \pm 6.8$ $10^{9} / \mathrm{L}$, and mean $\mathrm{SCr}$ was $72.8 \pm 28.6 \mathrm{umol} / \mathrm{L} ; 69$ patients $(75.0 \%)$ had hematuria, $17(18.5 \%)$ had proteinuria, 15 $(16.3 \%)$ had both proteinuria and hematuria, and 36 had oliguria/anuria (39.1\%). In the non-AKI group, the mean age was $36.1 \pm 17.4$ years, with 67 males and 116 females; the mean time of poisoning was $4.0(3.0) \mathrm{h}$, the mean amount of poison was $50.0(70.0) \mathrm{ml}$, the mean time to gastric lavage was 2.0 (2.0) h, the mean CRP level was $2.0(6.8) \mathrm{mg} / \mathrm{L}$, the mean peripheral blood leukocytes were $14.5 \pm 6.410^{9} / \mathrm{L}$, mean $\mathrm{SCr}$ was $62.4 \pm 17.0 \mathrm{umol} / \mathrm{L}$, and mean time from poisoning to blood purification was $6.0(3.5) \mathrm{h}$.

Between-group comparisons revealed that the differences were not statistically significant for any of the characteristics except for mean CRP level $(\mathrm{Z}=6.55, \mathrm{P}<0.001)$ and mean $\mathrm{SCr}$ $(t=-3.14, \mathrm{P}=0.002)$.

\subsection{Prognostic Outcomes of 275 Patients with Acute OP Poisoning}

The treatment prognosis of the 275 patients showed a cure rate of $72.4 \%(199 / 275)$ and a case fatality rate of $0.7 \%(2 / 275)$. The cure rate in the AKI group was $69.6 \%$ and the case fatality rate was $1.1 \%(1 / 92)$; the cure rate in the non-AKI group was $73.8 \%(135 / 183)$ and the case fatality rate was $0.5 \%(1 / 135)$. The difference in mortality between the two groups was not statistically significant $(Z=0.94, \mathrm{P}=0.34$ ), but the patients with complicating AKI had a long treatment time, long course of illness and high costs. Aside from 4 patients who required maintenance blood purification therapy, the remaining patients showed good outcomes for AKI, including 71 patients with normal clinical indicators, and 17 patients with mild azotemia. See Table 1. 
Table 1. Prognosis of treatment in the AKI and non-AKI groups after acute OP poisoning (n(\%)).

\begin{tabular}{llllll}
\hline Groups & Cured & Improved & Ineffective & Death & Total \\
\hline AKI $(92$ cases) & $64(69.6)$ & $23(25.0)$ & $4(4.3)$ & $1(1.1)$ & 92 \\
Non-AKI (183 cases) & $135(73.8)$ & $47(25.7)$ & $0(0.0)$ & $1(0.5)$ & 183 \\
Total & $199(72.4)$ & $70(25.5)$ & $4(1.4)$ & $2(0.7)$ & 275 \\
\hline
\end{tabular}

\subsection{Analysis of Factors Influencing AKI Onset}

By comprehensively taking into account the clinical data, sample size, whether the relationship between the independent variables and $\operatorname{logit} P(\mathrm{AKI}=1)$ was linear, and the goodness of model fit, the final model included the time of poisoning, amount of poison, time to blood purification, $\mathrm{SCr}$ and $\mathrm{CRP}$.
Among them, the time of poisoning, time to blood purification, and CRP were factors influencing AKI onset. The ORs and $95 \%$ confidence intervals (CIs) for time of poisoning and time to blood purification were $0.89(0.79,0.99)$ and 1.12 $(1.03,1.21)$, respectively. Since the relationship between CRP level and $\operatorname{logit} P(\mathrm{AKI}=1)$ was nonlinear, no interpretation was made of its OR value. See Table 2.

Table 2. Results of multivariate logistic regression analysis on AKI onset in acute OP poisoning.

\begin{tabular}{|c|c|c|c|c|}
\hline Factors & Wald X2 & $\mathbf{P}$ & OR & OR95\%CI \\
\hline Time of poisoning $(\mathrm{h})$ & 4.33 & 0.04 & 0.89 & $(0.79,0.99)$ \\
\hline Amount of poison $(\mathrm{ml})$ & 1.25 & 0.26 & 1.00 & $(1.00,1,01)$ \\
\hline Time to blood purification (h) & 7.37 & 0.01 & 1.12 & $(1.03,1.21)$ \\
\hline $\mathrm{SCr}^{2}(\mathrm{umol} / \mathrm{L})$ & 1.72 & 0.19 & 1.00 & - \\
\hline $\mathrm{CRP}(\mathrm{mg} / \mathrm{L})$ & 22.88 & $<0.001$ & 1.20 & - \\
\hline $\mathrm{CRP}^{2}(\mathrm{mg} / \mathrm{L})$ & 8.52 & 0.004 & 0.998 & - \\
\hline
\end{tabular}

Note: The original values of the independent variables were included in the model.

\section{Discussion}

OPs can be absorbed into the body via various routes, including the digestive tract, respiratory tract, skin and mucous membranes, where it travels through the blood and lymphatic systems to bind with a variety of tissue proteins in the body $[11,13]$, and is distributed to a variety of tissues and organs. Organophosphorus compounds are present in various forms, including as gases, droplets, aerosols or powders. In terms of daily life, dermal absorption is especially important because organophosphorus has a good lipid solubility, making it easier to be absorbed through the skin and mucous membranes.

After entering the body, OPs undergo two main metabolic processes: oxidation and hydrolysis. The oxidation pathway makes OPs more toxic through a series of reactions including deamination, dealkylation, reduction, side chain oxidation, and oxidative desulfurization, which transforms the original $\mathrm{P}=\mathrm{O}$ and its groups into $\mathrm{P}=\mathrm{O}$ groups with higher cholinesterase inhibiting activity. This is because the electronegativity of oxygen ions is greater than that of sulfur atoms, which increases the electropositivity of $\mathrm{P}$ atoms and facilitates the binding of OPs with the active site of cholinesterase, thereby exerting a stronger inhibition on cholinesterase activity, and increasing their toxicity to humans. Hydrolysis is a major mode of biotransformation, and its main role is to reduce the toxic effects of OPs. The relevant enzymes involved in the pathway include phosphatase, decarbonylase, carboxyl ester lipase, amidase, etc. $[14,16]$.

The acute toxicity of OPs is mainly achieved through the inhibition of cholinesterase activity, which includes acetylcholinesterase and butyrylcholinesterase [12]. These enzymes have different functions and actions depending on the location of the active site and the activating substrate. The series of toxic reactions caused by OP poisoning exert their toxic effects primarily through the inhibition of acetylcholinesterase, which inhibits the breakdown of the neurotransmitter acetylcholine into choline and acetate [13] and hence affecting the termination of signaling at cholinergic synapses. Acetylcholinesterase is widely present in the central and peripheral nervous systems, neuromuscular junctions, and red blood cells [14]. Therefore, OP poisoning is a serious and complex condition, and can endanger the patient's life in severe cases [15]. In recent years, related studies have pointed out that acute OP poisoning can lead to multiple organ dysfunction or even failure. Others have also found that AKI is not an isolated event, but can lead to multiple organ dysfunction, including the lung, heart, liver, intestine and brain. Possible mechanisms include neutrophil migration, cytokine expression and enhanced oxidative stress [17-19].

Toxic kidney injury due to acute OP poisoning often has an acute onset. Its clinical presentation is often divided into three phases: oliguric/anuric, polyuric, and non-oliguric acute renal failure. The oliguric/anuric phase mostly presents with water-electrolyte and acid-base imbalances, metabolite accumulation, and certain bleeding tendencies. The polyuric phase is when the 24-hour urine volume increases to more than $400 \mathrm{ml}$, and often manifests as a variety of electrolyte disorders. The non-oliguric phase presents as a maximum urine output of more than $800 \mathrm{ml}$ per day and a rising trend of blood creatinine; this phase progresses slowly and has a relatively good prognosis. For example, in our cohort of patients, the concomitant kidney damage did not increase 
mortality, which may be associated with aggressive and effective renal replacement therapy. Previous reports suggest that OP-induced AKI exhibit various clinical manifestations, for example, mild cases may simply have abnormal urinalysis with positive findings for protein or (and) occult blood, whereas severe cases may present with rapidly progressing oliguria/anuria and even require renal replacement therapy [20]. In this study, apart from four patients who required maintenance blood purification, all other patients showed good outcomes for AKI, no proteinuria and hematuria detected in urinalysis. Among whom, 71 patients had normal clinical indicators, and 17 patients had residual mild azotemia. However, patients with poisoning and complicating renal damage had a long course of disease. Overall, such patients showed good renal outcomes and prognosis, and the vast majority recovered their normal renal functions. In addition, more than 10 patients had renal biopsies that predominantly revealed acute tubulointerstitial lesions, which served as the pathological basis for good renal outcomes.

AKI caused by acute OP poisoning is a disease that is associated with multiple factors and has a complex pathogenesis [21]. The mechanisms leading to AKI are still unclear. However, some related studies have suggested that the possible mechanisms include: 1. damage and oxidation by oxygen free radicals; 2. inflammatory factors; 3. renal hemodynamic alterations; 4. induction of apoptosis; 5. excretion of toxins in the kidneys, causing peroxidative damage, damage to cell membranes, mitochondria and lysosomes, and other joint mechanisms. Recent evidence from basic research and clinical studies are gradually changing our understanding and opinion about the relationship between multiple organ dysfunction and AKI. These studies point to the important role of nephropathy in the overall process of disease onset, development, and outcome that starts from single organ dysfunction and progresses to multiple organ dysfunction syndrome. Our study showed that renal damage caused by acute OP poisoning was associated with a prolonged time of poisoning, prolonged time to blood purification, and high CRP levels. These findings suggest renal damage is related to the long retention time of the poison in the body and OP-induced inflammatory response. It also suggests that aggressive and early poison clearance and counteracting the inflammatory response, as well as the use of blood purification to clear the poison and inflammatory mediators, should serve as active and effective treatments.

Studies in China [22, 23] have also confirmed that there is a peak OP concentration in the early stage when it first enters the bloodstream and mostly exists in the free state. Thus, early detection, promptly seeking medical attention and shortening the time to medical consultation can reduce the absorption of poisons and improve the prognosis. Furthermore, the studies found that the reasonable implementation of blood purification therapy can accelerate the discharge of poisons, and was most effective when performed within 4 hours of poisoning. From our clinical data, we can conclude that patients with short poisoning time and early start of blood purification were able to have the poisons cleared as soon as possible, which resulted in a low incidence of AKI.

Studies have shown that severe OP poisoning is generally accompanied by systemic inflammatory response syndrome, which is due to the fact that OPs can activate immunologically active substances such as neutrophils after being absorbed, thus inducing the massive release of inflammatory mediators such as IL-1 $\beta$, IL-6 and IL-8 [24]. When the body is stimulated by microbial invasion or tissue damage, CRP synthesized by hepatocytes will increase dramatically within a few hours at the onset of inflammation and can peak at $48 \mathrm{~h}$. After which, CRP will gradually decrease to normal levels when the cause is eliminated, the lesion is diminished, and organ functions slowly recover. Radiation therapy, chemotherapy, or adrenocorticotropic hormones have little effect on CRP levels. It has been found that CRP activation mainly induces the immune response in three ways: Firstly, CRP can be broken down into monomers, which can serve as a defense mechanism for the body. Secondly, CRP can have a limiting effect on the potential damage caused by severe inflammation [25]. Finally, CRP is considered to be a pattern recognition receptor for innate immunity, and can effectively eliminate harmful substances [26]. The findings of Dalvi et al. $[27,28]$ showed that CRP is an independent risk factor in the assessment of prognosis for acute OP poisoning. Thus, it is an integral component of host defense and inflammation [29].

Therefore, in patients with acute OP poisoning, early administration of gastric lavage, suppression of systemic inflammatory response, and early blood purification therapy to accelerate the excretion of toxic substances from the body can slow down or even inhibit the onset of AKI and improve the prognosis of patients. Monitoring the changes of peripheral blood CRP can provide information on the patient's disease severity and guide clinical medication. However, this study has several shortcomings, including a small sample size, a retrospective design, and inadequacies in clinical observation. Therefore, the dynamic monitoring of renal histopathological data and sensitive indicators (biomarkers) of early kidney injury is still needed to better understand the clinical characteristics of AKI caused by OP poisoning, as well as its onset and development. This will enable the timely adoption of effective treatment plans, which will improve the patients' prognosis.

\section{Conclusion}

(1) Patients with acute OP poisoning and concomitant AKI have poorer prognosis.

(2) Prolonged time of poisoning, prolonged time to blood purification and increased CRP levels are risk factors for concomitant AKI.

\section{References}

[1] Kwong T C. Organophosphate pesticides: biochemistry and clinical toxicology [J]. Ther Drug Monit, 2002, 24 (1): 144 -149 . 
[2] Kishore GS, Krishnakanth KB. Poisoning severity score, APACHEII and GCS: Effective clinical indices for estimating severity andpredicting outcome of acute organophosphorus and carbamatepoisoning [J]. Journal of Forensic and Legal Medicine, 2009, (16): 239-247.

[3] Agostini M, Bianchin A: Acute renal failure from organophosphate poisoning $[\mathrm{J}]$ : a case of success with haemofiltration. Hum Exp Toxicol. 2003, 22: 165-167.

[4] Paudyal BP. Organophosphorus poisoning. JNMA J Nepal Med Assoc. 2008; 47: 251-258.

[5] Marraffa JM, Cohen V, Howland MA. Antidotes for toxicologicalemergencies: a practical review [J]. Am J Health Syst Pharm, 2012, 69 (3): 199-212.

[6] Lee FY, Chen WK, Lin CL, Lai CY, Wu YS, Lin IC, Kao CH. Organophosphate Poisoning and Subsequent Acute Kidney Injury Risk [J]. A Nationwide Population-Based Cohort Study. Medicine 2015; 94: 1-8.

[7] Pavan M. Acute Kidney Injury Following Paraquat Poisoning in [J]. IJKD 2013; 7: 64-66.

[8] Naqvi R. Snake-bite-induced Acute Kidney Injury [J]. J Coll Physicians Surg Pak 2016; 26: 517-520.

[9] Naqvi R. Scorpion Sting and Acute Kidney Injury [J].: Case Series from Pakistan. BJMMR 2015; 9: 1-6.

[10] Foxenberg RJ, McGarrigle BP, Knaak JB, Kostyniak PJ, Olson JR. Human hepatic cytochrome P450-specific metabolism of parathion and chlorpyrifos [J]. Drug Metab Dispos 2007; 35: 189-93.

[11] Goel A, Aggarwal P. Pesticide poisoning. N atl Med J India, 2007, 20 (4): 182-191.

[12] Dvir H, Silman I, HarelM, Rosenberry TL, Sussman JL. Acetylcholinesterase: from 3D structure to function $[\mathrm{J}]$. Chem Biol Interact 2010; 187: 10-22.

[13] Chowdhary S, Bhattacharyya R, Banerjee D. Acute organophosphorus poisoning [J]. Clin Chim Acta. 2014 4, (31): 66-76.

[14] Pakula AM, Skinner RA. Acute kidney injury in the critically ill patient: a current review of the literature. J Intensive Care Medicine. 2015 [Epub ahead of print].

[15] Li Y, Tse M L, Gawarammana I, et al. Systematic review of controlled clinicaltrials of gastric lavage in acute organophosphorus pesticide poisoning [J]. ClinToxicol (Phila), 2009, 47 (3): 179-192.

[16] Ventilation as a risk factor for acute kidney injury in the criticallyill: a systematic review and meta-analysis. Crit Care. 2013; 17: R98.
[17] Carey JL, Dunn C, Gaspari RJ. Central respiratory failure duringacute organophosphate poisoning. Respir Physiol Neurobiol. 2013; 189: 403-410.

[18] Liu L, Ding G. Effects of different blood purificationmethods on serum cytokine levels and prognosis in pa-tients with acute severe organophosphoruspesticide poi-soning [J]. Ther Apher Dial, 2015, 19 (2): 185-190.

[19] Shailesh KK, Pais P, Vengamma B, et al. Clinical and electrophsiological study of intermediate syndrome in patients with organophosphorous poisoning [J]. Assoc Physicians India, 1994. 42 (3): 451-453.

[20] Li A, Li W, Hao F, et al. Early stage blood purification for paraquatpoisoning: A multicenter retrospective study $[\mathrm{J}]$. Blood Purif, 2016, 42 (2): 93-99.

[21] Damman K, Valente MA, Voors AA, et al. Renal impairment, worsening renal function, and outcome in patients with heart failure: an updated meta-analysis [J]. Eur Heart J, 2014, 35 (7): 455-469.

[22] Van den akker JP, Egal M, Groenevld AB. Invasive mechanical ventilation as a risk factor for acute kindey injury in the critically ill: A systematic review and meta-analysis. Crit Care. 2013: 17: R98.

[23] Mittal G, Kumar N, Rawat H, etal. Development and clinical study of submicronic-atropine sulphate respiratory fluid as a novel organophosphorous poisoning antidote [J]. Drug Deliv, 2016, 23 (7): 2255-2261.

[24] Dalvi CP, Abraham P, Iyer SS. Correlation of electrocardiographic changes with prognosis in organophosphorus poisoning [J]. Postgrad Med, 1986, 32 (3): 115-119.

[25] Pepys M B, Hirschfield G M. C-reactive protein: A critical update [J]. J Clin Invest, 2003, 111: 1805-1812.

[26] C-reactive protein on leukocytes is fcgamma receptor II. J Exp Med, 1999, 190: 585-590.

[27] Lu J, Marjon K D, Marnell L L, et al. Recognition and functional activation of the human IgA receptor (FcalphaRI) by C-reactive protein. Proc Natl Acad Sci USA, 2011, 108: 4974-4979.

[28] Casas J P, Shah T, Hingorani A D, et al. C-reactive protein and coronary heart disease: A critical review. J Intern Med, 2008, 264: 295-314.

[29] Bharadwaj D, Stein M P, Volzer M, etal. The major receptor for C-reactive protein on leukocytes is fcgamma receptor II. J Exp Med, 1999, 190: 585-590. 